Н.Н. Рубцова ${ }^{1}$, Г.М. Борисов ${ }^{1,2}$, В.Г. Гольдорт ${ }^{1}$, Д.В. Ледовских ${ }^{1}$, А.А. Ковалев ${ }^{1}$, В.В. Преображенский ${ }^{1}$, М.А. Путято ${ }^{1}$, Б.Р. Семягин ${ }^{1}$

${ }^{1}$ Институт физики полупроводников им. А.В. Ржанова СО РАН, Новосибирск, Россия

${ }^{2}$ Новосибирский государственный университет, Новосибирск, Россия

\title{
ПОЛУПРОВОДНИКОВЫЕ ЗЕРКАЛА ДЛЯ СИНХРОНИЗАЦИИ МОД: НОВОЕ В ДИАГНОСТИКЕ И КОНСТРУКЦИИ
}

\begin{abstract}
Представлены результаты разработки для ближнего ИК-диапазона полупроводниковых зеркал с насыщающимся поглощением и их исследования в широком диапазоне интенсивностей излучения накачки методом накачка-зондирование. Результаты интерпретированы в рамках модели, учитывающей вклады в отражение фотогенерированных экситонов и свободных носителей заряда. Обсуждаются проблемы дальнейшего увеличения плотности сфокусированного рабочего излучения, допустимой для нормального функционирования и целостности зеркал, и способы их преодоления путем изменения конструкции.

Ключевые слова: квантовые ямы, быстродействующие полупроводниковые зеркала $\left(A_{3} B_{5}\right)$ с насыщением поглощения, пассивная синхронизация мод лазеров.
\end{abstract}

N.N. Rubtsova1, G.M. Borisov ${ }^{1,2}$, V.G. Goldort ${ }^{1}$, D.V. Ledovskikh', A.A. Kovalev', V.V. Preobrazhensky', M.A. Putiato', B.R. Semyagin ${ }^{1}$

${ }^{1}$ Institute of Semiconductor Physics A.V. Rzhanova SB RAS, Novosibirsk, Russian Federation

${ }^{2}$ Novosibirsk State University, Novosibirsk, Russian Federation

\section{SEMICONDUCTOR MIRRORS FOR MODE SYNCHRONIZATION: NEW IN DIAGNOSTICS AND DESIGN}

The article presents the results of the development for the near-infrared range of semiconductor mirrors with saturable absorption and their study in a wide range of pumping radiation intensities using the pump-probing method. The results are interpreted within the framework of a model that takes into account the contributions to the reflection of photo-generated excitons and free charge carriers. The problems of further increasing the density of focused working radiation that is permissible for normal functioning and the integrity of the mirrors, and ways to overcome them by changing the design are discussed.

Keywords: quantum wells, high-speed semiconductor mirrors (A3B5) with absorption saturation, passive mode locking of lasers. 
Получение коротких импульсов излучения при пассивной синхронизации мод в лазерах с широкой линией усиления известно, начиная с лазеров на красителях; эффект достигался путем подбора пары «усиливающий краситель-насыщающийся поглотитель» [1]. Такой подход получил новое звучание в связи с развитием полупроводниковых технологий [2]. Использование полупроводниковых зеркал с насыщающимся поглощением признано простым и надежным способом синхронизации мод для целого класса лазеров; имеются коммерчески доступные зеркала. К таким оптическим элементам предъявляют высокие требования: насыщаемое поглощение заданного уровня в определенной спектральной области усиления, быстрое просветление и восстановление поглощения, высокое отражение, низкий уровень ненасыщаемых оптических потерь во всей спектральной области усиления лазера. Кроме того, пользователи зачастую имеют в виду также высокую лучевую прочность по отношению к рабочему излучению, возможность длительной эксплуатации, низкую стоимость, независимость от импорта. Коммерчески доступные зеркала далеко не всегда отвечают всем перечисленным выше требованиям.

В нашей практике конструирования и изготовления полупроводниковых зеркал с насыщающимся поглощением для ближнего ИКдиапазона используются квантовые ямы с барьерами, структурированными наноразмерными вставками более узкозонного материала [3-5]. Изготовление структур методом молекулярно-пучковой эпитаксии (МПЭ) гарантирует высокую кристалличность образцов, которая контролируется в процессе роста. Материал квантовых ям не согласован по параметру решетки с материалом барьеров, непосредственно контактирующим с ямой, что создает механические напряжения; однако правильный выбор толщины слоев позволяет получать прочные структуры без дислокаций несоответствия. Количество квантовых ям регулирует глубину насыщающегося поглощения, а наноструктурирование барьеров позволяет достичь времени восстановления в несколько пикосекунд. Отражательная часть зеркал [3-5] была выполнена из пар четвертьволновых слоев арсенида галлия и арсенида алюминия. Исследование подобных зеркал было проведено [6] для интенсивностей рабочего излучения до 5,4 ГВт/см ${ }^{2}$ с центральной длиной волны 1035 нм. Техника измерений, основанная на одночастотном способе типа «накачка-зондирование», подробно описана в [7] и позволяет 
достигать чувствительности $5 \cdot 10^{-6}$ по относительному изменению отражательной способности. На рис. 1, а приведены формы кинетических кривых для минимальной и максимальной интенсивностей накачки, видно изменение формы кривых с ростом интенсивности. Хорошо видны участки быстрой релаксации (время релаксации $\tau_{1}$, соответствующее ионизации экситонов с фононами решетки) и медленной релаксации, определяющей быстродействие зеркала (время $\tau_{2}$, соответствующее электронно-дырочной рекомбинации). Подробное исследование формы кривых в рамках модели, учитывающей вклады в отражение фотогенерированных экситонов и свободных носителей заряда (их вклады видны на рис. 1, б для максимальной интенсивности накачки), показало, что интенсивность накачки влияет главным образом на амплитуды указанных вкладов. В таблице приведены для разных интенсивностей накачки времена релаксации экситонов $\tau_{1}$, электронно-дырочной рекомбинации $\tau_{2}$, время установления квазиравновесия фотогенерированных носителей заряда $\tau_{3}$, определяющее нарастание сигнала для электронно-дырочных пар, а также амплитудные вклады в отражение экситонов $A_{\text {exc }}$ и электронно-дырочных пар $A_{e h}$. Как видно из таблицы, временные параметры ( $\tau_{2}$ определяет быстродействие зеркал) остаются практически без изменения.

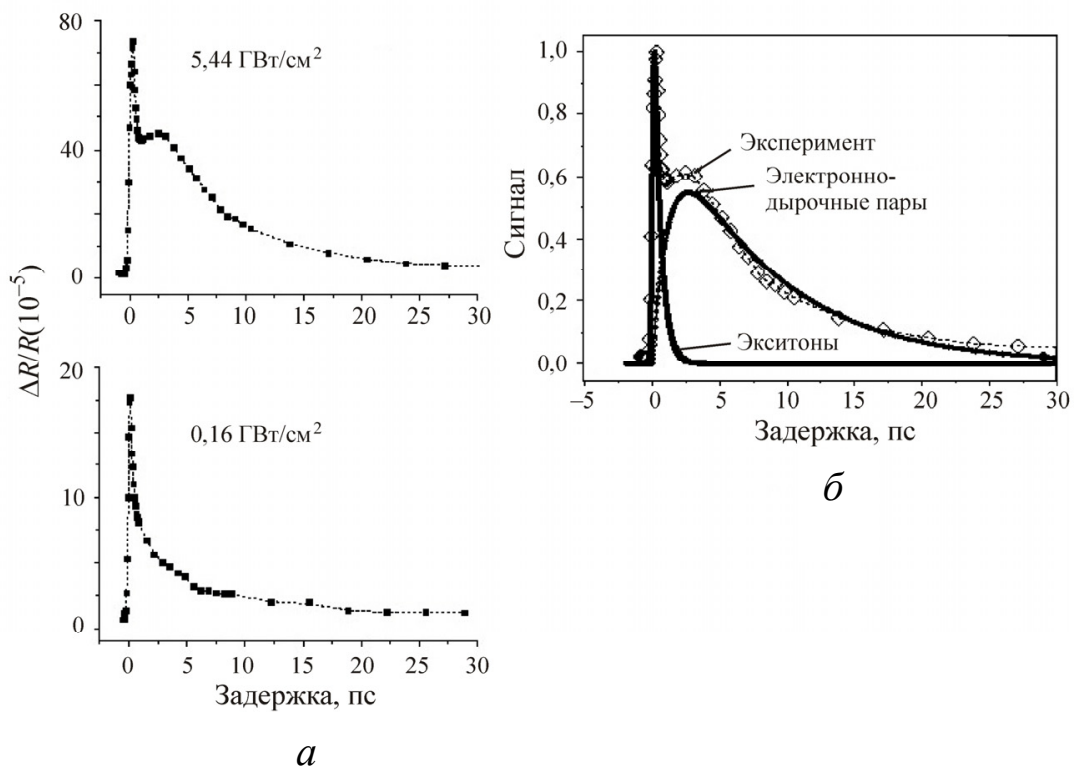

Рис. 1. Кинетика отражения кривых для низкой и высокой интенсивностей накачки $(a)$; вклады в отражение фотогенерированных экситонов и электронно-дырочных пар (б) 
Н.Н. Рубияова, Г.М. Борисов, В.Г. Гольдорт и др.

Временные параметры для разных интенсивностей накачки

\begin{tabular}{|c|c|c|c|c|c|}
\hline $\begin{array}{c}\text { Интенсивность, } \\
\text { ГВт/см }\end{array}$ & \multicolumn{2}{|c|}{ Временные параметры, пс } & \multicolumn{2}{c|}{ Амплитудные параметры, $10^{-4}$} \\
\cline { 2 - 6 } & $\tau_{1}$ & $\tau_{2}$ & $\tau_{3}$ & $A_{\text {exc }}$ & $A_{\text {eh }}$ \\
\hline 5,44 & 0,5 & 7,8 & 1,2 & 6,72 & 3,92 \\
\hline 2,73 & 0,45 & 7,8 & 0,8 & 3,23 & 1,47 \\
\hline 1,72 & 0,4 & 10 & 0,7 & 1,72 & 0,85 \\
\hline 0,54 & 0,35 & 10 & 0,7 & 1,82 & 0,56 \\
\hline 0,27 & 0,35 & 13 & 0,5 & 1,30 & 0,43 \\
\hline 0,16 & 0,38 & 12 & 0,4 & 1,62 & 0,50 \\
\hline
\end{tabular}

Из литературы, посвященной исследованиям полупроводниковых зеркал с насыщающимся поглощением, известно, что одной из причин, приводящих к нарушению работы зеркал и даже к их разрушению, может быть двухфотонное поглощение. Для разработанных нами структур для ближнего ИК-диапазона и для использованных энергий накачки не более 2 нДж роль двухфотонного поглощения была заметна лишь для структур, включающих массивные слои узкозонного материала (в нашем случае арсенида галлия), взаимодействующие с излучением накачки (т.е. не отделенные от квантовых ям отражательными слоями зеркала). В особенности это было заметно для массива квантовых ям, выращенных поверх подложки арсенида галлия без зеркального слоя [4].

Высокая отражательная способность зеркал [3-5] обеспечивалась выращиванием большого числа пар арсенида галлия и арсенида алюминия - материалов с хорошим согласованием по параметрам решетки. Специфика технологии МПЭ не позволяет обеспечить высокую однородность положения «стола» отражения в разных участках подложки. С целью избежать указанной неоднородности, а также с целью предотвратить возможный вклад двухфотонного поглощения в нарушение работы зеркала нами разрабатывается технология переноса слоя полупроводниковых квантовых ям на диэлектрическую подложку (широкозонный материал) в комбинации с диэлектрическим зеркалом. Пробная структура такого рода представляла собой выращенную на подложке GaAs ориентации (001) последовательность серии слоев. Это жертвенные слои InGaP и AlGaAs, позволяющие остановить травление после механического удаления большей части подложки; поверх них выращены 20 пар квантовых ям из материала 
$\mathrm{In}_{0,25} \mathrm{Ga}_{0,75} \mathrm{As}$ толщиной 27 монослоев, разделенных тонкими барьерами GaAs толщиной 3 монослоя, которые и служат насыщающимся поглотителем. Поверх этой структуры нанесено методом распыления в вакууме диэлектрическое зеркало из четвертьволновых слоев $\mathrm{SiO}_{2}$ и $\mathrm{ZrO}_{2}$. Затем сторона, закрытая диэлектрическим зеркалом, закреплена на сапфировой подложке, и затем проведено механическое и химическое удаление массива подложки из GaAs. Первый результат исследования кинетики структуры с полупроводниковым насыщающимся поглотителем и диэлектрического зеркала показан на рис. 2. Модельная кривая построена с параметрами $\tau_{1}=0,5$ пс и $\tau_{2}=3$ пс. Видна также «подкладка», интерпретированная как стационарное насыщение в массиве полупроводниковых жертвенных слоев, что отражает несовершенство существующей технологии.

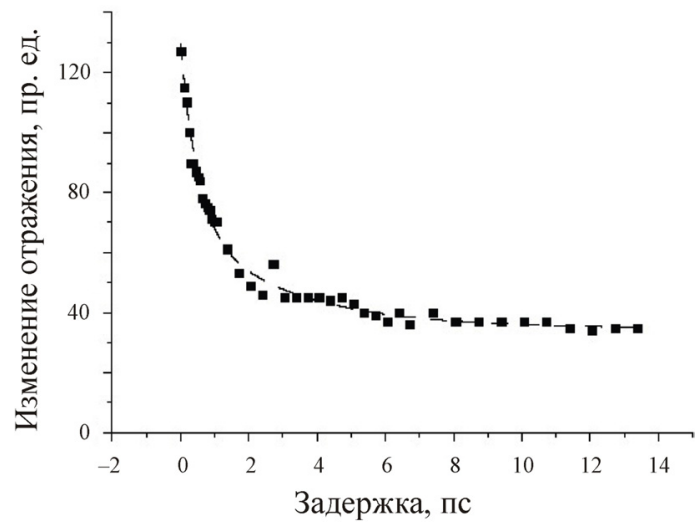

Рис. 2. Экспериментальная (квадраты) и модельная (штриховая линия) кинетика восстановления насыщенного отражения для полупроводникового насыщающегося поглотителя с диэлектрическим зеркалом (время восстановления $\tau_{2}=3$ пс)

В заключение отметим, что нами разработаны полупроводниковые зеркала с насыщающимся поглощением ближнего ИК-диапазона хорошего оптического качества, с низкими ненасыщаемыми потерями, достаточным быстродействием и стойкостью к рабочему излучению вплоть до интенсивности 5,4 ГВт/см². Зеркала были успешно испытаны в лазерах $\mathrm{Yb}: \mathrm{KYW}$ и $\mathrm{Yb}: \mathrm{KGW}[8,9]$. Начата работа по совершенствованию качества (в том числе по увеличению стойкости к интенсивному рабочему излучению ближнего ИК диапазона), удешевлению изделий и увеличению срока их службы. 
Авторы Г.М. Борисов и Д.В. Ледовских с благодарностью отмечают финансовую поддержку гранта РФФИ и субъекта РФ в рамках научного проекта № 18-42-543001\18.

\section{Список литературы}

1. Сверхкороткие световые импульсы / под ред. С. Шапиро. - М.: Мир, 1981. - 479 с.

2. Semiconductor saturable absorber mirrors (SESAMs) for femtosecond to nanosecond pulse generation in solid-state lasers / U. Keller, K.J. Weingarten, F.X. Kärtner, D. Kopf, B. Braun, I.D. Jung, R. Fluck, C. Hönninger, N. Matuschek, J. Aus der Au // IEEE J. Selected Topics in Quantum Electronics (JSTQE). - 1996. - Vol. 2. - P. 435-453.

3. 115 fs pulses from $\mathrm{Yb} 3+: \mathrm{KY}(\mathrm{WO} 4) 2$ laser with low loss nanostructured saturable absorber / A.A. Kovalyov, V.V. Preobrazhenskii, M.A. Putyato, O.P. Pchelyakov, N.N. Rubtsova, B.R. emyagin, V.E. Kisel', S.V. Kuril'chik, N.V. Kuleshov // Laser Physics Letters. - 2011. - Vol. 8, No. 6. - P. 431-435.

4. Reflectivity kinetics of saturable absorbers for laser mirrors / N.N. Rubtsova, G.M. Borisov, V.G. Gol'dort, A.A. Kovalyov, D.V. Ledovskikh // Laser Physics. - 2016. - Vol. 26. - P. 025001.

5. Fast mirrors for femtosecond passive mode-locked near-infrared lasers / N.N. Rubtsova, G.M. Borisov, D.V. Ledovskikh, A.A. Kovalyov, V.V. Preobrazhenskii, M.A. Putyato, B.R. Semyagin, V.E. Kisel', A.S. Rudenkov, N.V. Kuleshov, A.A. Pavlyuk // Laser Physics. - 2016. Vol. 26. - P. 125001.

6. Кинетика отражения полупроводникового быстродействующего зеркала / Г.М. Борисов, В.Г. Гольдорт, А.А. Ковалев, Д.В. Ледовских, Н.Н. Рубцова // Сибирский физический журнал. - 2017. - Т. 12, № 3. - C. 109-115.

7. Техника регистрации суб-пикосекундной кинетики отражения или пропускания / Г.М. Борисов, В.Г. Гольдорт, А.А. Ковалев, Д.В. Ледовских, Н.Н. Рубцова // ПТЭ. - 2018. - № 1. - С. 87-91.

8. High power efficient SESAM-mode-locked Yb:KGW bulk laser / V.E. Kisel', A.S. Rudenkov, A.A. Pavlyuk, A.A. Kovalyov, V.V. Preobrazhenskii, M.A. Putyato, N.N. Rubtsova, B.R. Semyagin, N.V. Kuleshov // Optics Letters. - 2015. - Vol. 40, No. 12. - P. 2707-2710.

9. Efficient high power femtosecond $\mathrm{Yb}^{3+}: \mathrm{KY}\left(\mathrm{WO}_{4}\right)_{2}$ laser / A.A. Kovalyov, V.V. Preobrazhenskii, M.A. Putyato, N.N. Rubtsova, B.R. Semyagin, V.E. Kisel', A.S. Rudenkov, N.V. Kuleshov, A.A. Pavlyuk // Laser Physics Letters. - 2015. - Vol. 12. - P. 075801. 


\section{References}

1. Sverkhkorotkie svetovye impulsy [Ultrashort light]. Ed. S. Shapiro. Moscow, Mir, 1981, 479 p.

2. Keller U., Weingarten K.J., Kärtner F.X., Kopf D., Braun B., Jung I.D., Fluck R., Hönninger C., Matuschek N., Aus der Au J. Semiconductor saturable absorber mirrors (SESAMs) for femtosecond to nanosecond pulse generation in solid-state lasers. IEEE J. Selected Topics in Quantum Electronics (JSTQE), 1996, vol. 2, pp. 435-453.

3. Kovalyov A.A., Preobrazhenskii V.V., Putyato M.A., Pchelyakov O.P., Rubtsova N.N., Semyagin B.R., Kisel' V.E., Kuril'chik S.V., Kuleshov N.V. 115 fs pulses from $\mathrm{Yb}^{3+}: \mathrm{KY}\left(\mathrm{WO}_{4}\right)_{2}$ laser with low loss nanostructured saturable absorber. Laser Physics Letters, 2011, vol. 8, no. 6, pp. 431-435.

4. Rubtsova N.N., Borisov G.M., Gol'dort V.G., Kovalyov A.A., Ledovskikh D.V. Reflectivity kinetics of saturable absorbers for laser mirrors. Laser Physics, 2016, vol. 26, pp. 025001.

5. Rubtsova N.N., Borisov G.M., Ledovskikh D.V., Kovalyov A.A., Preobrazhenskii V.V., Putyato M.A., Semyagin B.R., Kisel' V.E., Rudenkov A.S., Kuleshov N.V., Pavlyuk A.A.Fast mirrors for femtosecond passive mode-locked near-infrared lasers. Laser Physics, 2016, vol. 26, pp. 125001.

6. Borisov G.M., Goldort V.G., Kovalyev A.A., Ledovskikh D.V., Rubtsova N.N. Kinetika otrazheniya poluprovodnikovogo bystrodeystvuyushchego zerkala [ Reflection kinetics of a semiconductor high-speed mirror]. Sibirskiy fizicheskiy zhurnal, 2017, vol. 12, no. 3, pp. 109-115.

7. Borisov G.M., Goldort V.G., Kovalyev A.A., Ledovskikh D.V., Rubtsova N.N. Tekhnika registratsii sub-pikosekundnoy kinetiki otrazheniya ili propuskaniya [Sub-picosecond kinetics of reflection or transmission kinetics]. PTE, 2018, no. 1, pp. 87-91.

8. Kisel' V.E., Rudenkov A.S., Pavlyuk A.A., Kovalyov A.A., Preobrazhenskii V.V., Putyato M.A., Rubtsova N.N., Semyagin B.R., Kuleshov N.V. High power efficient SESAM-mode-locked Yb:KGW bulk laser. Optics Letters, 2015, vol. 40, no. 12, pp. 2707-2710.

9. Kovalyov A.A., Preobrazhenskii V.V., Putyato M.A., Rubtsova N.N., Semyagin B.R., Kisel' V.E., Rudenkov A.S., Kuleshov N.V., Pavlyuk A.A. Efficient high power femtosecond $\mathrm{Yb}^{3+}: \mathrm{KY}\left(\mathrm{WO}_{4}\right)_{2}$ laser. Laser Physics Letters, 2015, vol. 12, pp. 075801.

Получено 22.09.2018 